\title{
FLOSS Education: Long-Term Sustainability
}

\author{
Gregorio Robles $^{1}$, Jesús M. González-Barahona ${ }^{1}$, and Wouter Tebbens ${ }^{2}$ \\ 1 GSyC/LibreSoft, Universidad Rey Juan Carlos \\ $\{$ grex, jgb\}@libresoft.es \\ 2 Free Knowledge Institute \\ wouter@fki.org
}

\section{Summary}

Learning in any environment has undergone major changes in recent times. Many of these changes are closely related or are similar to processes or solutions found traditionally in Free/Libre and Open Source (FLOSS) environments: peer to peer relationships, telematic support, use of new technologies, etc. [1]

The purpose of this workshop is to bring together free software experts to discuss challenges that we face in the educational world at present and and that we will face in the future and how they can be undertaken from a FLOSS perspective. A public call for papers has been made resulting in various contributions, as follows:

- Utilization of OSS Virtual Machines for the Hands-on Training Environment

- Free and Open Source Software in Project Based Service Learning

- Learning through analysis of coding practices in FLOSS projects

- Sustainable Open Source Systems for Education and Research in Health Care: The Case of Cuba

- Portable Educational Portfolios

Based on this contributions and on the over 20 registered participants, a series of topics will be discussed, and conclusions and remarks will be collected and published. The contributions to and outputs of the workshop can be downloaded at the workshop website at http://libresoft.es/flossedu. This workshop is supported by the eMadrid network of Excellence (S2009 TIC-1650) and by the Free Knowledge Initiative.

\section{Reference}

1. Weller, M.: The Digital Scholar: How Technology is Transforming Scholarly Practice. Bloomsbury Academic, Basingstoke (2011) 